\title{
AUTOESTIMA Y VIOLENCIA DOMÉSTICA: INTERVENCIÓN PSICOTERAPÉUTICA
}

\section{SELF-ESTEEM AND DOMESTIC VIOLENCE: PSYCHOTHERAPEUTIC INTERVENTION}

\author{
Mayra del Rosario Villamarín Rodríguez ${ }^{1}$ \\ Mercedes Angélica García Pazmiño²
}

Recibido: 2019-02-05 / Revisado: 2019-03-16 / Aceptado: 2019-04-01 / Publicado: 2019-07-01

Forma sugerida de citar: Villamarín-Rodríguez, M. y García-Pazmiño, M. A. (2019). Autoestima y violencia doméstica: intervención psicoterapéutica. Retos de la Ciencia, 3(7), pp. 48-55. https://doi.org/10.53877/rc.3.7.20190701.05

\section{RESUMEN}

La autoestima es confiar en sí mismo y en sus propias capacidades para enfrentar los retos de la vida y llegar a la autorrealización. Por otro lado, la violencia doméstica es una agresión de tipo físico, psicológico, social, patrimonial o sexual que afecta la autoestima y la salud mental de la víctima, llevándola a un estado de indefensión y desajuste psicosocial. En este estado de vulnerabilidad, la intervención psicoterapéutica grupal, permite reconocer la problemática en un ambiente de solidaridad y cooperación, desarrolla conductas asertivas y habilidades sociales afectivas. El objetivo del estudio fue relacionar la efectividad de la terapia grupal para el mejoramiento de la autoestima en mujeres víctimas de violencia doméstica. Metodológicamente el enfoque cuantitativo, correlacional; diseño de corte preexperimental longitudinal. La recolección de datos fue con las escalas de Coopersmith y Satisfacción con el Tratamiento Recibido, en una muestra no probabilística de 16 mujeres en condición de violencia doméstica del Centro de Atención Integral. El registro inicial de autoestima fue bajo y medio. Posterior a la intervención se elevó la autoestima sustancialmente. La hipótesis probó una correlación positiva entre variables. En conclusión, las mujeres intervenidas subieron la autovaloración y asertividad en el manejo en las relaciones interpersonales.

Palabras clave: autoestima, violencia doméstica, intervención psicoterapéutica, terapia grupal.

\section{ABSTRACT}

Self-esteem is trusting in oneself and one's own abilities to face the challenges of life and reach self-realization. On the other hand, domestic violence is an aggression of a physical, psychological, social, patrimonial or sexual type that affects the self-esteem and mental health of the victim, leading to a state of defenselessness and psychosocial imbalance. In this state of vulnerability, the group psychotherapeutic intervention, allows to recognize the problem in an atmosphere of solidarity and cooperation, develops assertive

\footnotetext{
1 Psicóloga Clínica. Docente del Instituto Superior Tecnológico Central Técnico. Ecuador. E-mail: mdvillamarin@uce.edu.ec

${ }^{2}$ Doctora en Ciencias de la Salud y del Trabajo (Ph. D). Profesora en la Facultad de Psicología de la Universidad Central del Ecuador. Ecuador. E-mail: magarciap1@uce.edu.ec
} 
behaviors and affective social skills. Objective: To relate the effectiveness of group therapy in the improvement of self-esteem in women victims of domestic violence. Quantitative, correlational approach; design of longitudinal pre-experimental cutting. Through the application of the Coopersmith scale and satisfaction with the treatment received in a nonprobabilistic sample of 16 women in domestic violence status of the Comprehensive Care Center. Initial registration of the low and medium self-esteem level (7:10). After the intervention 8:10 women raised their self-esteem; 7:10 showed a high level of satisfaction with the treatment received. The hypothesis proved a positive correlation between variables. Intervened women gained self-assessment and assertiveness in the management of interpersonal relationships.

Key words: self-esteem, domestic violence, psychotherapeutic intervention, group therapy

\section{INTRODUCCIÓN}

La violencia contra la mujer ha existido a lo largo de la historia y con mucha más presencia en sociedades patriarcales. La violencia doméstica es considerada como un problema de salud pública de primer orden, según la Organización Mundial de la Salud. Desde la literatura, la violencia doméstica se entiende como las agresiones que se producen en el ámbito privado, en donde el agresor tiene una relación con la víctima, tomando en cuenta la reiteración de los actos violentos y la situación de dominio del agresor que utiliza la violencia para someter y controlar a la víctima. (Fernández, 2003) De la extensa revisión literaria, los datos estadísticos reflejan una creciente problemática social el tema de la violencia, de cualquier forma, que esta se manifieste. Por ello, se encuentra necesario generar procesos de atención dirigidos a la comunidad de mujeres afectadas, ya que las secuelas que conlleva la violencia doméstica afectan directamente a la autoestima de quien la padece, volviéndola un ser vulnerable, indefenso y silencioso.

Al respecto, el Instituto Nacional de Estadísticas y Censos (INEC, 2011), refleja los índices estadísticos en una muestra de 18.800 viviendas con mujeres de 15 años y más en 24 provincias a nivel nacional urbano y rural: 6 de cada 10 mujeres han vivido algún tipo de violencia de género, la forma de violencia más recurrente es la psicológica $(53,9 \%)$, seguida por la física $(38 \%)$, sexual $(25,78 \%)$ y patrimonial $(16,7 \%)$. Por otro lado, las mujeres ecuatorianas que han vivido algún tipo de violencia por parte de sus parejas o ex-parejas corresponden al $48,7 \%$.

Según el psicoterapeuta Branden (1995) la autoestima es la confianza depositada en la capacidad individual para enfrentarse a los desafíos de la vida y conseguir su felicidad, puesto que una autoestima plena conlleva a tener una vida más significativa. Así también, se considera a la autoestima como la percepción valorativa y confiada de sí misma, que motiva a la persona a manejarse con propiedad, manifestarse con autonomía y proyectarse satisfactoriamente en la vida (Mezerville Zeller, 1995).

Por otro lado, una persona que cuenta con una deficiente autoestima se caracteriza por mantener un estado de insatisfacción consigo misma, es hipersensible a la crítica, indecisa todo el tiempo por miedo a equivocarse, hay un deseo excesivo de complacer a los demás, exagera la magnitud de sus errores y tiene tendencia a la depresión generalizada (García, 1990).

Frente a la necesidad de reconstrucción, empoderamiento y mejoramiento de la autoestima como recurso indispensable para el desarrollo humano en un contexto de violencia, las terapias grupales se convierten en una alternativa de solución, para romper los círculos vicios de la violencia. La terapia grupal comprende un proceso que desplaza el problema individual hacia lo grupal, en donde a través de la interacción y las narraciones de los miembros les permite darse cuenta que otros también tienen similares problemas 0 conflictos, de tal forma poder reconstruir las realidades de los participantes, ya que es un espacio terapéutico que da la apertura a ver, pensar y sentir una visión social diferente a la conocida en el espacio familiar (Serebrinsky, 2012). 
La terapia grupal tiene su origen con Hersey Pratt (1872-1956), médico internista del Hospital General de Massachusetts, observó que el proceso grupal parecía tener un efecto terapéutico y que también ayudaba a disminuir el tiempo que debía destinar a cada enfermo de forma individual. A su vez, podía promover un ambiente de solidaridad y cooperación. El autor propone encuentros en cinco sesiones semanales de una hora de duración, al inicio no se examinaban los síntomas de los asistentes, sino que se realizaban ejercicios de relajación, de imaginación y conversaciones de orientación médica convenientes para los pacientes. A medida que el tiempo transcurre aparecen otros investigadores que abordan la temática, como Scheidlinger y Slavson (citados en Portillo Díaz, 2000) al inferir que la "terapia de grupo tiene como objetivo terapéutico la conveniencia del grupo, bajo un proceso social en el que el profesional especializado, utiliza la interacción emocional en el grupo, para reparar la salud mental" (p.40).

De igual forma, (Carneiro, Teodor y Chicone, 2006), demostraron que la baja autoestima es un factor desencadenante de situaciones de exclusión, abandono y maltrato frente a la situación de amenaza vivida con la pareja con consecuencias negativas para la calidad de vida de la víctima. Todos los seres humanos tienen necesidades de afecto, protección y seguridad en el entorno familiar y en la comunidad. Así también, necesitan reconocer su amor propio, confianza, reconocimiento, apreciación y admiración; sin embargo, cuando la insatisfacción de ellas por procesos de violencia a los cuales son sometidas, genera sentimientos de inferioridad e impotencia, provocando fracasos en su funcionalidad y despertando procesos patológicos variados

Durante el mismo año, López, Apolinaire, Array y Moya (2006) realizaron un estudio para analizar el nivel de autovaloración en las mujeres que han sido maltratadas, y concluye que las mujeres que poseen una autovaloración positiva se sienten más confiadas, capaces, aceptadas y respetadas con un sentido real de autodeterminación para manejar sus relaciones de pareja. Este estudio se llevó a cabo en una muestra de 40 mujeres violentadas a quienes se aplicó el Inventario de Coopersmith; el método que utilizó fue de corte observacional analítico y concluye en que hay una relación significativa entre las mujeres violentadas y la autovaloración.

Respecto a las ventajas de la terapia grupal en grupos de mujeres que han sido víctimas de violencia de género (Ayats, Cirici y Soldevilla, 2008), en el cual se evalúo la efectividad de una intervención grupal cognitivo-conductual en mujeres violentadas, realizado con siete grupos de mujeres, bajo un método experimental, con sesiones periódicas de 90 minutos, aplicando técnicas de autocontrol, asertividad y reconstrucción. Los resultados indican mejoras significativas respecto a la depresión e histeria; determinando que la terapia grupal es un proceso efectivo para el tratamiento con mujeres que han sido violentadas.

Algo semejante ocurre con la propuesta de investigación de Hernández (2013), enfocada a la atención psicológica grupal para las mujeres que padecen secuelas de la violencia de género, la cual tiene por objetivo diseñar un programa con protocolos que permitan atender las necesidades específicas de las mujeres afectadas. Este estudio utiliza el método documental y técnicas de tipo vivencial aplicado a seis participantes, obteniendo como resultado la creación del programa "Volver a ser Yo", aclarando que el trabajo con poblaciones vulnerables representa un reto, porque se deben cuidar los objetivos y planes que se desea cumplir con sus participantes.

Posteriormente, se realizó otro estudio similar, pero esta vez el proceso de la terapia grupal se aplicaría a mujeres deprimidas víctimas de violencia intrafamiliar en una muestra no probabilística de 8 mujeres. El objetivo era determinar si un programa de terapia grupal disminuye los niveles de depresión en las participantes, para ello se usó un diseño cuasiexperimental y estadístico, y se aplicó el Inventario de Beck, instrumento que permitió comprobar que el $88 \%$ de las mujeres evaluadas presentaban algún nivel de depresión, señalando que la violencia intrafamiliar representa un alto riesgo para la salud mental de la mujer, y que por esta razón la terapia grupal es una herramienta terapéutica que ayuda a disminuir los niveles de depresión, siempre y cuando se asista permanentemente (Lemus, 2014). 
Más adelante, otro estudio relevante realizado por Lafuente Bolufer (2015) refiere que la intervención grupal con mujeres víctimas de violencia de género mediante el taller "Creciendo Juntas", su objetivo es reforzar la autoestima y seguridad en las participantes, un grupo de 10 mujeres españolas entre 28-55 años de edad, utilizó una metodología abierta y participativa, con técnicas de dinamización grupal, herramientas como el Inventario de Coopersmith facilitan la medición de la autoestima. Como resultado se logró establecer lazos de apoyo entre las asistentes, permitiendo a las mujeres manifestar sus necesidades respecto a su autoestima, seguridad y asertividad.

Así también, desde un enfoque cualitativo construccionista, se analiza la psicoterapia grupal en procesos de separación de pareja, cuyo objetivo es conocer los cambios realizados en las historias personales de cuatro participantes adultos ante la separación de su pareja, como resultado se obtuvo cambios positivos en sus relaciones personales, las técnicas de la narrativa según el presente estudio, permiten que las conversaciones terapéuticas colectivas sean usadas para la deconstrucción de verdades y mandatos sociales así como para la reconstrucción de la historia personal. (Saénz y, 2015)

En la última década, se ha detectado mediante estudios, la temprana violencia que se puede desarrollar en las relaciones de noviazgo entre adolescentes, para ello se utilizó un método cuantitativo descriptivo, con una muestra de 600 estudiantes que tenían relaciones de noviazgo, se les aplicó el cuestionario de autoestima de García, el Inventario de Rosemberg y con el fin de identificar las características de la violencia asociadas con el nivel de autoestima, los instrumentos arrojaron resultados indicando que el 93,2\% de los adolescentes viven violencia en sus relaciones y que la identifican y consideran como un proceso normal. (Rodríguez, González y Hernández, 2011)

El presente estudio se posicionó teóricamente en el modelo terapéutico de Rogers (1940), quien elaboró una propuesta de terapia no directiva, centrada en la persona, cuyo postulado sostiene que la energía del individuo se relaciona con la tendencia al crecimiento, salud y desarrollo; cada persona toma decisiones necesarias. Por otro lado, Cloninger (2003) manifestó que hay que abordar las emociones en primer plano, el intelecto y las vivencias en segundo plano.

\section{METODOLOGÍA}

El presente estudio se realizó con un enfoque cuantitativo, el mismo que permite medir fenómenos investigados (Hernández y Cols, 2014). Diseño correlacional de corte preexperimental longitudinal. La población, con una muestra no probabilística de tipo por conveniencia, consistente en 16 mujeres con vivencia de conflictos de violencia intrafamiliar, que acuden al Centro de Atención Integral. Se aplicó una encuesta sociodemográfica; la escala de autoestima de Coopersmith y la Escala de satisfacción con el tratamiento recibido Cres-4. La intervención psicoterapéutica con enfoque grupal, fue precedida de una primera evaluación y posterior a ella una segunda toma. Éticamente el estudio no implica riesgo para las participantes.

La planificación psicoterapéutica, abarcó un período de nueve sesiones, una sesión por semana con una duración de tres horas, por un lapso de tres meses consecutivos; constó de cuatro momentos: inclusión, confrontación, afecto y cierre. La inclusión, comienza con la formación del grupo, en donde los miembros necesitan ser aceptados e incluidos por los demás. Se establecen límites, el nivel de compromiso y de predisposición. La confrontación de los miembros del grupo ya se establece de algún modo, empieza a surgir la necesidad de reconocer la problemática, asumir responsabilidades y orientar el comportamiento a la toma de decisiones. El afecto, surge los fenómenos grupales, como la cohesión, la integración, apoyo y confianza. Es la descripción intensa de las emociones, las mismas que pueden ser negativas o positivas, en ocasiones puede resultar ser perturbador y difícil para los individuos y finalmente, el cierre, La retroalimentación, se da mediante una comunicación constante entre el facilitador y los miembros del grupo, de alguna forma resulta a veces complicado el hecho de aceptar la opinión de los demás, para ello se necesita manejar adecuadamente los contenidos del mensaje. 


\section{RESULTADOS Y DISCUSIÓN}

Del análisis estadístico descriptivo de la población de mujeres violentadas que asistieron a la terapia grupal en el Centro Atención Integral. Se encuentran en una edad mínima de 19 y máxima de 57 años, lo que indica que la violencia se ejerce en cualquier grupo etario; asimismo el rango de edad prevalente es de 30 a 40 años. Respecto a su estado civil los datos indican que 5:10 mujeres están casadas o en unión libre en iguales porcentajes, 4:10 son solteras y sólo 1:10 está divorciada (Tabla 1).

Tabla 1

Estado civil según edad

\begin{tabular}{|c|c|c|c|c|c|c|c|}
\hline \multicolumn{2}{|c|}{} & \multicolumn{6}{c|}{ Estado civil } \\
\cline { 3 - 8 } & Casada & Divorciada & Soltera & $\begin{array}{c}\text { Unión } \\
\text { libre }\end{array}$ & Total & $\%$ \\
\hline \multirow{5}{*}{ Edad } & $19-29$ & 0 & 0 & 2 & 1 & 3 & 19 \\
\cline { 2 - 8 } & $30-39$ & 3 & 1 & 1 & 2 & 7 & 44 \\
\cline { 2 - 8 } & $40-49$ & 1 & 0 & 2 & 1 & 4 & 25 \\
& $50-57$ & 0 & 1 & 1 & 0 & 2 & 13 \\
\cline { 2 - 8 } & Total & 4 & 2 & 6 & 4 & 16 & 100 \\
\cline { 2 - 8 } & Porcentaje & 25 & 13 & 38 & 25 & 100 & \\
\hline
\end{tabular}

Fuente: Villamarín y García (2019)

En la tabla 2 se puede observar que 4:10 mujeres víctimas de violencia doméstica, tienen instrucción secundaria, la mitad de ellas no tienen una labor remunerada, factor que conlleva a que su situación económica sea inestable, constituyendo un factor de riesgo para la victimización.

Tabla 2

Instrucción académica y ocupación laboral

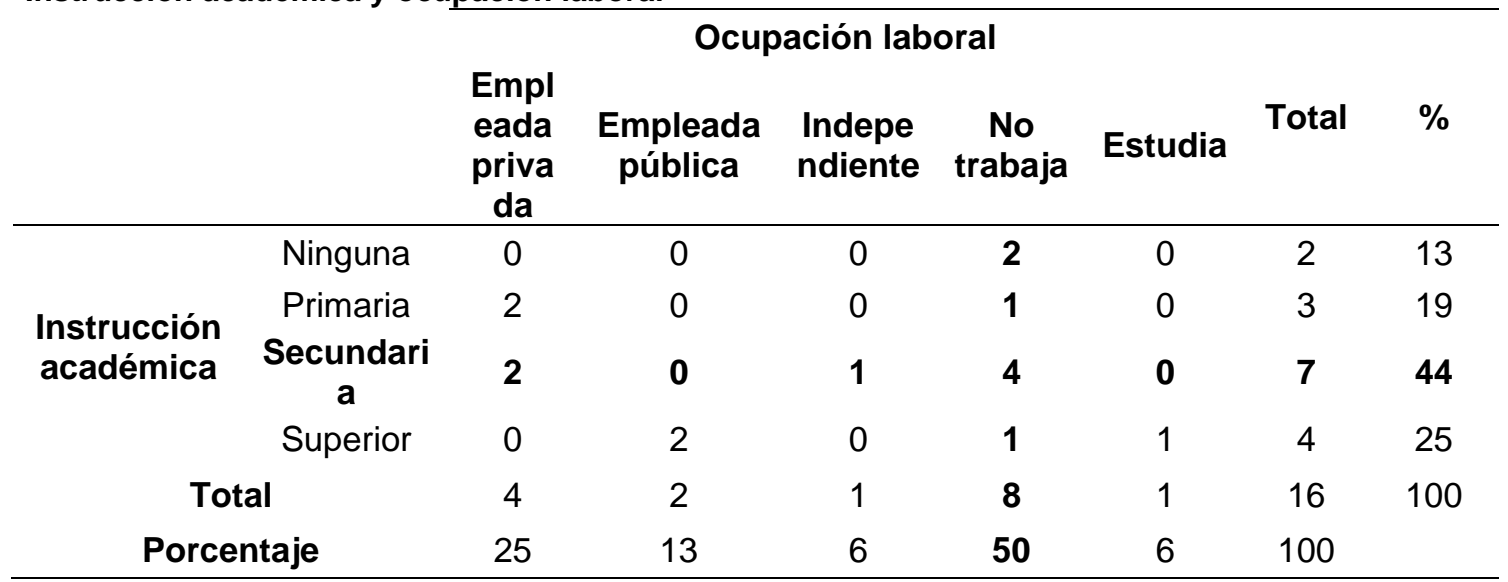

Fuente: Villamarín y García (2019)

En relación a la victimización y la denuncia del hecho, se refleja que todas las mujeres participantes en el estudio han vivenciado violencia doméstica; es decir, que fueron sometidas reiteradamente a actos violentos, malos tratos y situaciones de dominio por parte del agresor, quien utiliza la violencia para someter y controlar a la víctima. Alrededor de 7:10 mujeres denunciaron los hechos de violencia y fueron remitidas a terapia grupal desde las Unidades Judiciales (Tabla 3). 
Tabla 3

Denuncia y victimización

\begin{tabular}{|c|l|c|c|c|}
\hline \multicolumn{2}{|c|}{} & Victimización & \multirow{2}{*}{$\%$} & \multirow{2}{*}{ Total } \\
\cline { 3 - 5 } \multicolumn{2}{|c|}{} & $\mathrm{Si}$ & & \\
\hline \multirow{2}{*}{ Denuncia } & $\mathrm{Si}$ & 12 & 75 & 12 \\
\cline { 2 - 5 } & $\mathrm{No}$ & 4 & 25 & 4 \\
\hline \multicolumn{2}{|r|}{ Total } & 16 & 100 & 16 \\
\hline
\end{tabular}

Fuente: Villamarín y García (2019)

De la valoración de los niveles de autoestima según la Escala de Coopersmith, en la primera toma se identificó que 7:10 mujeres presentaron un nivel de autoestima bajo y medio bajo; y 3:10 autoestima entre medio alto y alto. En la segunda toma, después de la intervención psicoterapéutica 8:10 mujeres elevaron la autoestima medio alta y alta, mejorando la autovaloración y seguridad consigo mismas. Resultados que se encuentran en relación directa con el nivel de compromiso para acudir a las sesiones de terapia grupal y a su vez se corroboran con (García, 1990) al indicar que la baja autoestima genera trastornos psicológicos de origen neurótico que dificultan el desarrollo de la vida personal, individual y social de la víctima (Tabla 4).

Tabla 4

Nivel de autoestima en mujeres víctimas de violencia

\begin{tabular}{cccccc}
\hline & \multicolumn{3}{c}{ Test 1 } & \multicolumn{2}{c}{ Test 2 } \\
\cline { 2 - 6 } & Categoría & Frecuencia & Porcentaje & Frecuencia & Porcentaje \\
& Bajo & $\mathbf{3}$ & $\mathbf{1 9}$ & $\mathbf{0}$ & $\mathbf{0}$ \\
Nivel de & Medio bajo & 8 & 50 & 3 & 23 \\
Autoestima & Medio alto & 3 & 19 & 4 & 31 \\
& Alto & $\mathbf{2}$ & $\mathbf{1 3}$ & $\mathbf{6}$ & $\mathbf{4 6}$ \\
\cline { 2 - 7 } & Total & 16 & 100 & 13 & 100 \\
\hline
\end{tabular}

Fuente: Villamarín y García (2019)

En relación a la satisfacción con la terapia grupal recibida 8 de cada10 mujeres manifestaron un nivel alto de satisfacción, coincidente con el número de mujeres que elevaron el nivel de autoestima (Tabla 5). Algo semejante ocurrió con la propuesta de investigación de Hernández (2013) que fue diseñar un programa con protocolos que permitieron atender las necesidades específicas de las mujeres afectadas. Aplicó técnicas de tipo vivencial aplicado a seis participantes, concluyó que el trabajo con poblaciones vulnerables representa un reto.

Tabla 5

Escala global de satisfacción con el tratamiento recibido CRES-4

\begin{tabular}{lcc}
\hline \multicolumn{1}{c}{ Categoría } & Frecuencia & Porcentaje \\
\hline Nivel bajo de satisfacción & 2 & 15 \\
Nivel medio de & 2 & 15 \\
satisfacción & 9 & 69 \\
Nivel alto de satisfacción & 13 & 100 \\
Total &
\end{tabular}

Fuente: Villamarín y García (2019)

La hipótesis se probó con la prueba de correlación de Pearson donde $r=0.88$, comprobó, una correlación positiva entre variables, porque a medida que una variable 
aumente la otra también; es decir, que la terapia grupal optimizó el nivel de autoestima de las mujeres víctimas de violencia doméstica (Tabla 6).

Tabla 6

Correlación de terapia grupal y autoestima

Terapia grupal * Autoestima

\begin{tabular}{|c|c|c|c|}
\hline & & $\begin{array}{l}\text { Terapia } \\
\text { grupal }\end{array}$ & Autoestima \\
\hline \multirow[t]{3}{*}{ Terapia grupal } & $\begin{array}{l}\text { Correlación } \\
\text { de Pearson }\end{array}$ & 1 & ,881 \\
\hline & $\begin{array}{l}\text { Sig. } \\
\text { (bilateral) }\end{array}$ & &, 000 \\
\hline & $\mathrm{N}$ & 16 & 16 \\
\hline \multirow[t]{3}{*}{ Autoestima } & $\begin{array}{l}\text { Correlación } \\
\text { de Pearson }\end{array}$ & ,881 & 1 \\
\hline & $\begin{array}{l}\text { Sig. } \\
\text { (bilateral) }\end{array}$ &, 000 & \\
\hline & $\mathrm{N}$ & 16 & 16 \\
\hline
\end{tabular}

Fuente: Villamarín y García (2019)

\section{Conclusiones}

Las mujeres en situación de vulnerabilidad debido a que fueron víctimas de violencia doméstica, son jóvenes adultas y están casadas o en unión libre, en su mayoría tienen una instrucción de segundo nivel; más de la mitad no tienen una actividad económica remunerada, lo cual constituye un factor de riesgo que conlleva a la dependencia económica de sus parejas y aceptar pasivamente la violencia y generando un estado de indefensión volviéndose en ocasiones cómplices de la violencia recibida, como lo demuestra el presente estudio el $25 \%$ de mujeres que no denuncian e invisibilizan a la violencia.

En relación al nivel de autoestima, se estableció que al inicio de la terapia grupal el $70 \%$ de las mujeres violentadas tenían autoestima en un nivel bajo y medio bajo; y el $30 \%$ restante mantiene su autoestima entre medio alto y alto; una vez que reciben la terapia grupal el $80 \%$ elevaron el nivel autoestima, seguridad y valoración; mientras que el $30 \%$ aún necesitan procesos de fortalecimiento para elevar el autoestima y salir del estado de indefensión.

El estudio comprobó la efectividad de la terapia grupal en el fortalecimiento de la autoestima en mujeres que han vivenciado violencia doméstica, generando seguridad y respeto hacia sí mismas; y además, lograron mantener un adecuado manejo de su relación de pareja, interfamiliar, rompiendo el círculo de la violencia doméstica, mejorando las relaciones interpersonales.

\section{AGRADECIMIENTOS}

Se deja constancia de agradecimiento al Centro de Atención Integral "Tres Manuelas" por la aquiescencia y colaboración para realizar el estudio. A las mujeres que se atrevieron a romper el silencio y el círculo vicioso de la violencia doméstica.

\section{REFERENCIAS BIBLIOGRÁFICAS}

Ayats, M., Cirici Amell, R. y Soldevilla, J. (2008). Grupos de terapia para mujeres víctimas de violencia de género. Sociedad Española de Psiquiatría Biológica 15(2), 29-34. Recuperado

de https://dialnet.unirioja.es/servlet/articulo?codigo=2658688\&info=resumen\&idioma=SPA

Branden, N. (1995). Los seis pilares del autoestima. México: Editorial Planeta. Recuperado de 
https://www.researchgate.net/publication/31658693_Los_Seis_pilares_de_la_autoestim a_el_libro_definitivo_sobre_la_autoestima_por_el_mas_importante_especialista_en_la materia_N_Branden_tr_por_Jorge_Vigil_Rubio

Carneiro, L., Teodor, G. y Chicone, G. (2006). Autoestima de las mujeres que sufrieron violencia. Revista Latino-Americana de Enfermagem 14(5), 695-701. https://doi.org/10.1590/S0104-11692006000500009

Cloninger, S. (2003). Teorías de la Personalidad. México: Pearson Educación de México S.A. Tercera Edición.

Fernández, M. y cols. (2003). Violencia Doméstica. Madrid: Ministerio de Sanidad y Consumo.

Recuperado

https://www.mscbs.gob.es/ciudadanos/violencia/docs/VIOLENCIA_DOMESTICA.pdf

García, J. (1990). Autoestima y salud. Recuperado de http://www.alezeia.org/seminarios/wpcontent/uploads/utoestimsa-y-Salud.pdf

Hernández Sampieri y cols. (2014). Metodología de la investigación. Journal of Chemical Information and Modeling, 53(9), 1-589. Recuperado de https://doi.org/10.1017/CBO9781107415324.004

Hernández, X. (2013). Propuesta de atención psicológica grupal para mujeres que padecen secuelas de violencia de género 2-5. Recuperado de http://repositorio.iberopuebla.mx/handle/20.500.11777/193

Instituto Nacional de Estadísticas y Censos. (2011). Encuesta Nacional de las Relaciones Familiares y Violencia de Género contra las Mujeres. Recuperado de http://www.ecuadorencifras.gob.ec/documentos/webinec/Estadisticas_Sociales/sitio_violencia/presentacion.pdf

Lafuente, E. (2015). Mujeres víctimas de violencia de género: taller "creciendo juntas. Universidad Jaume I de Castellón. Recuperado de http://repositori.uji.es/xmlui/bitstream/handle/10234/161071/TFM_Lafuente

Bolufer Eugenia.pdf?sequence $=1 \% 0 \mathrm{~A}$

Lemus, A. (2014). "Aplicación y propuesta de un programa de Terapia Grupal a mujeres deprimidas, víctimas de violencia intrafamiliar, que asisten al Servicio Nacional de la Mujer (SERNAM. Chile: Universidad Rafael Landívar. Recuperado de http://biblio3.url.edu.gt/Tesario/2014/05/22/Lemus-Amanda.pdf\%0A

López, L., Apolinaire, J. J., Array, M. y Moya, A. (2006). Autovaloración en mujeres víctimas de violencia de pareja. MediSur 4(1), 9-12. Recuperado de http://www.redalyc.org/articulo.oa?id=180019846003\%0A

Mezerville, G. (1995). El Aprendizaje de la Autoestima como proceso educativo y terapéutico. Index, 17(1) 5-13. Recuperado de https://revistas.ucr.ac.cr/index.php/educacion/article/view/12688/11940

Portillo, I. (2000). Bases de la Terapia de Grupo. México: Editorial Pax. Recuperado de https://es.scribd.com/doc/197762167/Gerald-Corey-Teoria-y-practica-de-la-terapiagrupal

Rodríguez, M., González, C. y Hernández, M. (2011). Violencia de Género y Nivel de Autoestima en las Relaciones de Noviazgo de los Adolescentes. Desarrollo Cientif Enferm, 19(1), 25-29. Recuperado de http://www.indexf.com.roble.unizar.es:9090/dce/19pdf/19-025.pdf\%5Cnhttp://www.indexf.com.roble.unizar.es:9090/new/cuiden/extendida.php?cdid=668342_1

Saénz, A., y Trujano, P. (2015). Análisis de la psicoterapia grupal construccionista. Psicología lberoamericana, 23(2), 77-85. Recuperado de http://www.redalyc.org/pdf/1339/133944231009.pdf\%0A

Serebrinsky, H. (2012). Psicoterapia de Grupo. AJAYU 10(2), 132-135. Recuperado de http://www.redalyc.org/pdf/4615/461545462001.pdf 